\title{
Linéarité et consonnes latentes
}

\section{Patrick Sauzet}

\section{OpenEdition}

Journals

Édition électronique

URL : http://journals.openedition.org/rlv/1214

DOI : $10.4000 /$ rlv. 1214

ISSN : 1958-9239

\section{Éditeur}

Presses universitaires de Vincennes

\section{Édition imprimée}

Date de publication : 1 mai 1999

Pagination : 59-86

ISSN : 0986-6124

\section{Référence électronique}

Patrick Sauzet, "Linéarité et consonnes latentes », Recherches linguistiques de Vincennes [En ligne], 28| 1999, mis en ligne le 09 septembre 2005, consulté le 21 avril 2019. URL : http:// journals.openedition.org/rlv/1214; DOI : 10.4000/rlv.1214 


\title{
Patrick SAUZET \\ Université Paris 8
}

\section{LINÉARITÉ ET CONSONNES LATENTES}

\begin{abstract}
RÉSUMÉ
Soit l'hypothèse que la linéarité n'est pas constatée et utilisée, mais bien construite par la syllabation dans les morphèmes, à partir d'une accessibilité différenciée des segments. D'une part cela permet de poser que la phonologie produit aussi pour partie l'ordre des morphèmes, et se trouve ainsi à l'origine de la morphologie et du mot. D'autre part cela autorise à poser, de manière dérivée ou lexicale, des morphèmes dont le contenu phonique est plus connexe qu'une concaténation ou, inversement, dont le contenu phonique est discontinu. Utilisant cette dernière possibilité, on caractérisera la déficience des consonnes latentes du français, non comme un flottement (statut plus problématique qu'il ne semble), mais comme une dissociation. Une telle analyse permet d'envisager, outre la latence constante (« petit, petit-t-ami, petitesse »), la latence variable (« grand, grande, grand-t-ami », « vert, verte, verdure »), ainsi que d'autres faits de supplétisme (« beau, bel ») et de variabilité finale («sec, sèche »).
\end{abstract}

\section{MoTS-CLÉS}

Linéarité, consonnes latentes, liaison, syllabation, français. 
Je me propose ici de revenir sur la question des consonnes latentes et de la liaison en français, en relation avec un ensemble de propositions touchant la linéarité dans la grammaire. J'expose ces propositions ${ }^{1}$ avant d'examiner ce qu'elles peuvent apporter au traitement des consonnes latentes.

\section{Linéarité en phonologie}

La linéarité a traditionnellement un statut différent en phonologie et dans les autres composantes de la grammaire (syntaxe, morphologie). En syntaxe la linéarité est dérivée de l'organisation hiérarchique finale. Il en va de même en morphologie, si on suppose une organisation structurale des morphèmes associée à leur ordre linéaire, ce que Lisa Selkirk appelle une « syntaxe des mots. »C'est ce que posent les modèles de Lieber $(1980,1992)$ Selkirk (1982), Williams (1981) ou de Baker (1988) pour n'en citer que quelques-uns ${ }^{2}$. Cela est vrai aussi, indirectement, de la «morphologie distribuée » qui manipule des morphèmes, bien que le contenu phonique de certains, ou de tous, ne soit introduit que secondairement (Halle \& Marantz, 1993; Marantz, 1997). La simple terminologie courante, qui évoque «pré-fixes » ou « suf-fixes », est une stipulation directe de l'ordre des éléments.

$\mathrm{Au}$ contraire, on considère habituellement que la linéarité est, en phonologie, toujours déjà donnée. La phonologie exploite un ordre linéaire qui la précède : celui que construit la syntaxe ou la morphologie, celui, lexicalement fixé, des segments dans les morphèmes. Cette asymétrie apparaît de manière plus éclatante à partir du moment où on assigne à la phonologie la tâche de construire aussi de la structure, syllabique ou métrique. On peut légitimement supposer (c'est en fait l'hypothèse la moins marquée) que cette structure repose sur les mêmes propriétés formelles (le format X' pour citer un candidat probable et souvent proposé) à travers les composantes de la grammaire. Mais, d'un composant à l'autre, cette structure commune se trouve dans une relation très différente à la linéarité : tantôt elle la produit, tantôt elle ne fait que l'interpréter. C'est parce qu'il dépend structuralement du verbe qu'un syntagme nominal objet le suit en français. Inversement c'est parce qu'elle se trouve suivre la voyelle « $\mathrm{O}$ » que « $\mathrm{r}$ » est interprété comme une coda dans « sortie » : on construit les syllabes sur une suite de segments dépourvue de structure mais déjà linéaire. (1b) dérive de (1a) :

$$
\text { a. /s rrti/ } \rightarrow \text { b. } \quad\left(\mathrm{s}\left({ }_{\mathrm{R}} \mathrm{J} \mathrm{r}\right)\right)\left(\mathrm{t}\left({ }_{\mathrm{R}} \mathrm{i}\right)\right)
$$

Cette situation pourrait légitimement être imputée au caractère globalement interprétatif de la phonologie si la syntaxe ou la morphologie produisaient toute la linéarité que la phonologie interprète. Mais la phonologie traite d'abord la linéarité interne des morphèmes et cette linéarité est supposée donnée lexicalement.

Le coût de cette présupposition est volontiers sous-estimé parce qu'il est notationnellement nul. Soit le morphème et mot « joli », on note /zoli/ la suite des 
segments qui le composent. Grâce aux conventions reçues de l'écriture, la simple juxtaposition à droite sur la même ligne (ou au début de la ligne suivante) note la relation «successeur unique de ». Si on note $\mathrm{S}(\mathrm{x}, \mathrm{y})$ cette relation « y est le successeur unique de $\mathrm{x}$ », (2b) [où les accolades suspendent la pertinence linéaire de l'agencement typographique] explicite (2a) :
a. /ろoli/
b. $\{3, \jmath, 1, i\}$ tels que $S(3, \jmath), S(\supset, 1), S(1, i)$.

Une notation équivalente consiste à faire référence à la série numérique comme pure expression de la relation «successeur unique de ». Ce qui donne :

$$
\{(1,3)(2, \supset)(3, \supset),(4, \mathrm{i})\}
$$

où le morphème est un ensemble de couples associant un nombre qui donne l'ordre et un contenu phonique. Les représentations, utilisées à partir de Vergnaud (1982), Kaye \& Lowenstamm (1984), Levin (1985), où les segments sont rattachés à des positions sont des équivalents de (3). Les positions pures valent pour des nombres qui instruisent l'ordre des segments.

Il est peu satisfaisant d'avoir à supposer que les morphèmes comportent une caractérisation numérique, la phonologie ne faisant jamais référence à un décompte du nombre des segments. Elle ne compte que par l'établissement de dépendances locales (rimes branchantes, pieds bornés), ou par mise en parallèle d'unités récurrentes qui ne teste que la coïncidence de la cardinalité, voir Cornulier (1982: 58).

Fondamentalement la linéarité consiste dans l'utilisation du temps. On fait se suivre des caractérisations qui ne peuvent être simultanées à cause des limitations de production et de perception du canal vocal-auditif qu'utilisent les langues naturelles (langues signées mises à part). Autant ces contraintes s'imposent à la production du langage, autant elles ne sont pas des propriétés nécessaires du savoir instantané du locuteur, considéré hors de sa mise en œuvre ${ }^{3}$.

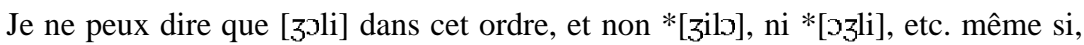
comme on le sait, les segments idéalisent une succession phonique dont les corrélats articulatoires et acoustiques réels sont largement chevauchants ${ }^{4}$. Rien n'implique que ce morphème en tant qu'il fait partie du savoir d'un locuteur comporte la même successivité ou son équivalent (que constitue l'ordre numérique). Autrement dit, la linéarité n'est pas une propriété nécessaire (ni probable) des objets lexicaux et du savoir du locuteur.

Reste la pertinence de l'ordre linéaire dans la réalisation. Les paires minimales fondées sur l'ordre des segments : [mal] « mal(le)» [lam] « lame », [ote] « ôté » [eto] « étau », etc. l'illustrent bien. Toutefois, la seule implication de cette pertinence est que quelque chose dans la caractérisation lexicale d'un morphème assure l'expression linéaire constante de son contenu, et distingue éventuellement deux morphèmes de contenu segmental par ailleurs identique. 
Rien n'implique qu'il s'agisse là de la linéarité elle-même, déjà présente et établie. Que [lam] s'oppose à [mal] interdit de considérer que leur forme sousjacente est simplement l'ensemble $\{\mathrm{a}, 1, \mathrm{~m}\}$. Mais il ne s'ensuit pas de là qu'il faille présupposer /mal/ et /lam/ prélinéarisés.

On dispose d'une structure phonologique qui permet de construire l'ordre linéaire : la syllabe. Si l'on sait que dans l'ensemble ci-dessus l'élément (m) est coda, on ne peut plus construire, (a) étant noyau, qu'une rime $\left[_{R} a m\right]$ et de là une syllabe $\left.\left[{ }_{S} 1{ }_{R} a m\right]\right]$. Cette syllabe, ayant une structure, peut avoir un ordre : l'attaque y précède le noyau, que la coda suit.

Si la syllabation peut produire l'ordre, quelle information supposer présente qu'elle puisse convertir en successivité ? La spécification directe d'un statut syllabique s'accommode mal avec la variabilité de ce statut dans un morphème soumis à dérivation : $[(\mathrm{lam})]$ « lame »vs $[(\mathrm{la})(\mathrm{mel})]$ « lamelle ${ }^{5}$. Elle s'accommode mal aussi avec le Principe de l'attaque maximale, PAM, en anglais MOP Maximal Onset Principle, voir Clements \& Keyser (1981 : 19), qui implique que la syllabation soit calculée sur une forme et non instruite de manière spécifique pour chaque syllabe: «joli » est syllabé [(३⿰)(li)], et [(३つl)(i)] est exclu, bien que la syllabe ( 3 l) n'ait rien de mal formé. Par ailleurs, les paires minimales dues à des différences de statut syllabique («paye » [pej], « pays » [pei], « oui » [wi], « ouille » [uj]) sont plutôt marginales, en sorte qu'on peut supposer que ce n'est pas par la syllabation que ces formes diffèrent abstraitement (et tout au plus qu'une caractérisation lexicale exceptionnelle de la syllabation est possible).

Si l'on suppose la linéarité produite par un processus de structuration phonologique, et d'abord par la syllabation, l'hétérogénéité nécessaire du matériau phonique des entrées lexicales doit être une différence d'accessibilité à ce processus. Traitant d'abord ce qui est le plus accessible, le processus de linéarisation-syllabation lui donne une position différente de celle du matériau qu'il traite ensuite.

Admettons que la syllabation (puis la mise en place des structures accentuelles qui la prolonge) établit, à partir du matériau lexical, un objet hiérarchique linéairement réalisable, de façon continue dans une direction, de gauche à droite ou de droite à gauche. De là trois hypothèses : soit une des deux directions est universellement utilisée pour construire la linéarité, soit le choix de la direction est variable. L'hypothèse d'une direction unique universelle paraît beaucoup plus plausible. Il faudrait supposer des corrélats très repérables à une variation affectant un paramètre aussi profond et fondamental de la faculté de langage. Reste à savoir quelle est la direction universelle. Je retiens l'hypothèse d'une construction universelle de la linéarité opérant de droite à gauche, c'est-àdire de manière rétrospectivement rétrograde. En d'autres termes le matériau phonique réalisé final est considéré comme issu des spécifications les plus accessibles.

Ce choix semble souhaitable pour diverses raisons. La construction régressive rend plus naturel le PAM, en tant que maximalisation de la structure construite. Le critère de maximalisation est utilisé pour déterminer le sens de la 
syllabation par Itô $(1986: 163 ; 1989)$ qui doit bien spécifier que la formation de la syllabe nucléaire (« core syllable ») échappe à ce critère (Itô, $1986: 10)^{6}$. L'hypothèse régressive aussi permet d'analyser la linéarisation de morphèmes comme un prolongement de la linéarisation à l'intérieur d'un même morphème, une extension essentielle qui sera évoquée ci-dessous ${ }^{7}$.

Notons enfin que cette hypothèse régressive exige (alors que la conception progressive ne fait que la permettre) une production phonique organisée en blocs anticipés : on ne commence à réaliser un objet phonologique linéarisé qu'en l'ayant défini jusqu'à sa fin (quitte à renoncer à le produire effectivement). Cela semble conforme aux fonctionnements réels (voir l'article de Sophie Wauquier ici-même). L'anticipation, ainsi rendue nécessaire, autorise le calage de l'accentuation sur la fin de mot et divers processus régressifs : assimilations, harmonies, élision, etc.

Supposons qu'un morphème est un signe saussurien ${ }^{8}$ qui unit d'emblée une caractérisation phonique et un contenu non phonique : propriétés sémantiques et caractérisation catégorielle associée. Il est assez naturel d'admettre que c'est à partir de ces dernières propriétés que se fait l'accès au contenu phonique, autrement dit que c'est à partir du sens qu'un morphème est normalement mobilisé. Par commodité je représenterai désormais le plus souvent tout le contenu non phonique d'un morphème, dont l'existence m'importe ici plus que l'organisation interne, par la seule indication de la catégorie (Nom, Verbe, Adj.). Entre les diverses propriétés d'un morphème et les unissant, je pose une relation unique, symétrique et illimitée, l'attenance, simple adjacence non orientée ${ }^{9}$ (nécessairement non orientée puisque préexistant à toute successivité). Je note explicitement l'attenance par des traits diversement disposés selon la commodité typographique, ainsi dans les exemples de (4). L'attenance unit dans un morphème des spécifications homogènes comme hétérogènes. Un élément phonique attenant à la partie non phonique d'un morphème est plus accessible qu'un autre (qui n'est attenant qu'à d'autres éléments phoniques qui médiatisent sa relation au contenu non phonique).

La linéarité présupposée est donc remplacée par une accessibilité différenciée du contenu phonique des morphèmes, définie par les modalités de l'association au sein de ceux-ci de spécifications hétérogènes, autrement dit par la réalisation spécifique de l'arbitraire du signe. Celle-ci n'est plus la mise en relation globale de deux faces du signe, mais l'articulation fine de propriétés élémentaires ${ }^{10}$. Cela peut se représenter comme en (4) pour le mot « sel » [sel] :

a. $\quad$ s-e-1-Nom

b. Nom-1-E-s

c) Nom 
Les formes notées en (4) a, b ou c, et toutes les dispositions spatiales qu'on voudra imaginer sont strictement équivalentes. Dans toutes, le segment ${ }^{11} / 1 /$ est le plus accessible. Les configurations données en (5) ou d'autres semblables sont aussi possibles :
a) $\mathrm{s}-\mathrm{l}-\mathrm{Nom}$ 11
$\varepsilon$
b) $\mathrm{s}-1$
$1 / 1$
$\varepsilon-$ Nom
c) $\mathrm{s}-\mathrm{l}$
/1
$\varepsilon-$ Nom

En fait c'est une question empirique de déterminer si une configuration comme (4) ou comme (5) est plus adéquate.

On peut supposer, par exemple, que (4) est possible mais plus marqué que (5a) [si la connectivité est privilégiée] ce qui implique, les deux formes se linéarisant de manière identique, que le dernier format sera toujours choisi. On peut à l'inverse supposer que des formes comme (5), présentant des possibilités de chemins ambigus d'un élément à l' autre sont exclues parce qu'ininterprétables. Qu'il soit clair surtout que si l'on utilise des formes comme (4), elles ne sont pas d'avance définies comme linéaires. Elles sont « quasiment linéaires », en ce sens qu'elles n'ont que deux extrémités et qu'il n'y a qu'un chemin entre elles : en remplaçant l'attenance par une relation de succession on en fait des objets linéaires alors que ce n'est pas le cas des formes de (5). Ce qui est décisif, c'est qu'elles n'ont pas un tel format (à supposer qu'elles doivent l'avoir) par stipulation directe : seulement par restriction induite, au sens de Dell (73:205), c'est-à-dire par un effet des procédures d'interprétation qui s'appliquent à elles. J'utiliserai des formes comme (4) sans plus discuter ici, parce qu'elles permettent une exposition plus simple. De plus, ce choix n'exclut pas de poser aussi des formes lexicales radicalement non linéaires dans des cas où cela apparaît empiriquement nécessaire (Sauzet, 1994a : 174).

Tout mécanisme qui respectant l'accessibilité construit des syllabes (par hypothèse vers la gauche) peut produire de la linéarité à partir de formes comme (4).

J'utilise la structure de syllabe ${ }^{12}$ en (6), de format X', et le mécanisme de syllabation en (7).

(6) Syllabe

$$
\left((\mathrm{s})\left(\varepsilon_{\mathrm{s}}{ }^{\circ}(1)\right)=((\text { attaque })(\text { noyau }(\text { coda })))=((\text { adjonction })(\text { tête }(\text { complément })))\right.
$$

La tête syllabique (ici $\varepsilon^{\circ}$ ) gouverne la coda. Les constituants autres que la tête, et en particulier la coda, sont des projections maximales, c'est-à-dire en un sens des syllabes réduites, conformément à la récursivité qu'implique la théorie $\mathrm{X}$ ', ici utilisée dans une version à deux niveaux significatifs de projection. 
(7) a. Algorithme de syllabation

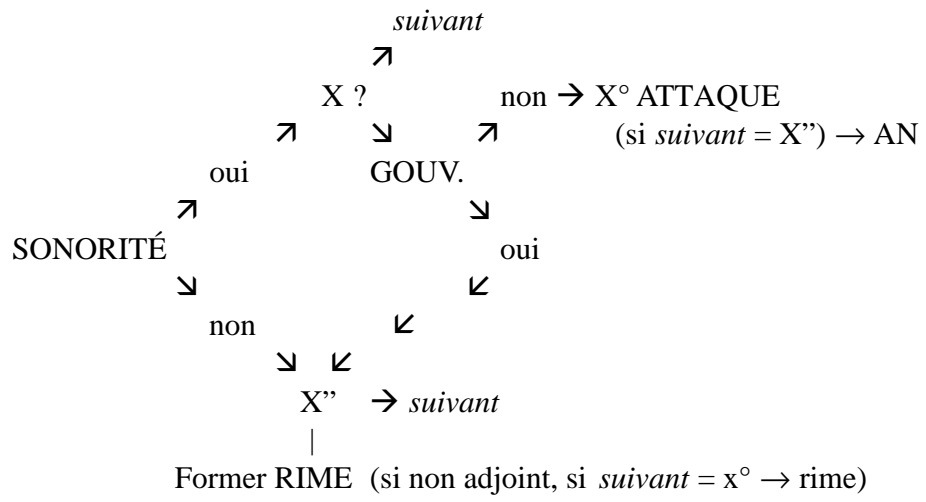

b. Explication de l'algorithme :

- SONORITÉ : le degré de sonorité du segment est-il compatible avec le statut de tête?

- Gouv. : le segment est-il localement gouvernable ? Oui, si un segment suivant est $\mathrm{X}^{\circ}$ (tête) et plus sonore. Non si suivant est soit moins sonore, soit X" (projection maximale).

- suivant : passer au(x) segment(s) attenant(s) (et non encore parcourus) et reprendre l'algorithme à SONORITÉ.

- AtTAQue : si un segment est identifié tête $\left(\mathrm{X}^{\circ}\right)$, former avec suivant s'il est $\mathrm{Y}$ " (et dégénéré) une chaîne $\mathrm{Y}^{\prime} \mathrm{X}_{\mathrm{Y}}{ }^{\circ}$ (où le noyau porte l'indice de l'attaque). La condition "et dégénéré » produit une langue sans attaque branchante, les langues qui connaissent les attaques branchantes peuvent former une attaque avec une syllabe non dégénérée dont le noyau est moins sonore que le noyau de la syllabe principale : $\left(\left(\mathrm{t} \mathrm{r}^{\circ}\right)\left(\mathrm{i}^{\circ}\right)\right.$ «tri »; notons que la condition de moindre sonorité de l'attaque ne joue pas dans le cas des attaques non branchantes ${ }^{13}$.

Remarque : on sera amené plus loin à poser que l'attaque est formée, non seulement avec un segment attenant, mais plus généralement avec un segment accessible, c'est-à-dire avec un segment dont $\mathrm{X}^{\circ}$ n'est pas lui-même le suivant. Ce segment peut être attenant à $\mathrm{X}^{\circ}$ mais aussi, comme on le verra, être le plus accessible dans un autre morphème ou dans un autre fragment du même morphème (au contenu phonique discontinu).

- RIME : si une projection maximale Z" n'est pas adjointe et que suivant est une tête $X^{\circ}$, former $\left(X^{\circ}\left(Z^{\prime}\right)\right)$. [N.B. le fait que $X^{\circ}$ ait reçu une attaque en adjonction n'affecte pas sa capacité à gouverner.] 
RIME est absent des langues CVCV. Il n'est pas exclu que RIME permette de gouverner des projections maximales complexes et que la récursivité syllabique soit donc parfois non triviale (Sauzet, 1994b : 48).

- $X$ ? quand un segment n'est identifié ni comme tête $\left(X^{\circ}\right)$ ni comme projection maximale (X") le processus d'identification se dédouble : il reste actif pour le segment non identifié, noté $X$ ?, jusqu'à ce que GOUV soit décidable, et s'enclenche parallèlement dans le segment suivant (suivant). Les deux flèches issues d'un point de l'algorithme ne sont donc des disjonctions que si les aboutissants sont incompatibles (« oui » et « non »).

Les dérivations suivantes illustrent le fonctionnement de l'algorithme (7) :

(8)

\begin{tabular}{|l|l|l|}
\hline «thé » & t-e-Nom & \\
\hline & $(\mathrm{t})-\mathrm{e}^{\circ}$ & $\begin{array}{l}-\mathrm{e} \text { assez sonore, non gouvernable donc tête }: \mathrm{e}^{\circ} \\
-\mathrm{t} \text { n'est pas assez sonore donc non-tête }:(\mathrm{t}) .\end{array}$ \\
\hline & $\left((\mathrm{t})\left(\mathrm{e}_{\mathrm{t}}^{\circ}\right)\right)$ & - coindiciation et adjonction de l'attaque. \\
\hline
\end{tabular}

(9)

\begin{tabular}{|l|l|l|}
\hline «bail » & b-a-i-Nom & \\
\hline & b-a-i ${ }^{?}$ & $-\mathrm{i}$ assez sonore, gouvernable ? \\
\hline & b-a ${ }^{\circ}-(\mathrm{i})$ & $\begin{array}{l}-\mathrm{a} \text { assez sonore, non gouvernable donc } \mathrm{a}^{\circ} \text {; par suite } \mathrm{i} \\
\text { set gouvernable, donc (i). }\end{array}$ \\
\hline & $\left((\mathrm{b})\left(\mathrm{a}_{\mathrm{b}}{ }^{\circ}(\mathrm{i})\right)\right)$ & $\begin{array}{l}- \text { coindiciation et adjonction de l'attaque ; formation la } \\
\text { rime. }\end{array}$ \\
\hline
\end{tabular}

(10)

\begin{tabular}{|l|l|l|}
\hline «sel » & s-E-l-Nom & \\
\hline & s-E- (l) & -1 ne peut être tête $\left(\right.$ en français $\left.{ }^{14}\right) ; 1$ est gouvernable. \\
\hline & $(\mathrm{s})-\varepsilon^{\circ}-(1)$ & $\begin{array}{l}- \text { E peut être tête et n'est pas gouvernable localement, } \\
\text { s ne peut être tête. }\end{array}$ \\
\hline & $\left((\mathrm{s})\left(\mathrm{E}_{\mathrm{s}}^{\circ}(\mathrm{l})\right)\right)$ & $\begin{array}{l}\text { - coindiciation et adjonction de l'attaque ; formation de } \\
\text { la coda. }\end{array}$ \\
\hline
\end{tabular}


(11)

\begin{tabular}{|c|c|c|}
\hline \multirow[t]{5}{*}{ « joli » } & 3-o-1-i-Adj & \\
\hline & $3-0-(1)-i^{\circ}$ & $\begin{array}{l}\text { - i peut être tête et n'est pas gouvernable ; } \\
1 \text { ne peut être tête et est gouvernable. }\end{array}$ \\
\hline & $3-9-\left((1)\left(i_{1}^{\circ}\right)\right)$ & - coindiciation et adjonction de l'attaque. \\
\hline & $(3)-\bigcirc^{\circ}-\left((1)\left(i_{1}^{\circ}\right)\right)$ & $\begin{array}{l}\text { - o peut être tête et n'est pas gouvernable } \\
\text { localement, s ne peut être tête. }\end{array}$ \\
\hline & $\left((3)\left(\mathfrak{o}_{3}^{\circ}\right)\right)\left((1)\left(i_{1}^{\circ}\right)\right)$ & $\begin{array}{l}\text { - coindiciation et adjonction de l'attaque ; formation } \\
\text { de la coda. }\end{array}$ \\
\hline
\end{tabular}

En (11), une fois formée la première syllabe, si le matériau phonique n'est pas épuisé, une nouvelle syllabe est adjointe à gauche de la première : $\left(3^{\circ}\right)$ est adjoint à $\left(\mathrm{li}^{\circ}\right)$. Je considère la structure où les syllabes sont successivement adjointes à gauche de la première syllabe formée (la syllabe finale) comme la structure suprasyllabique par défaut. Elle correspond à un accent final implicite ou réalisé. Des propriétés accentuelles marquées ou lexicales déterminent diverses déviations à partir de ce schéma de défaut (construction de constituants). En français la déviation est minimale, l'accent final (ou l'absence d'accent) réalisant la proéminence structurale par défaut. Seule une syllabe finale contenant schwa peut se trouver intégrée dans un objet final plus complexe que la syllabe ordinaire (légitimée par l'accent).

$$
\begin{aligned}
& \left(_{\text {no }}\left(\mathrm{e}^{\circ}\right)\left(_{\text {no }}\left(\mathrm{tu}^{\circ} \mathrm{r}\right)\left(_{\mathrm{no}} \mathrm{no}^{\circ}\right)\right)\right) \text { «étourneau » } \\
& \left(_{\mathrm{r} \varepsilon}(\mathrm{ka})\left(_{\mathrm{r} \varepsilon}(\mathrm{ta})\left(_{\mathrm{r} \varepsilon}(\mathrm{rak})(\mathrm{t} \ni)\right)\right)\right) \text { 《 cataracte » }
\end{aligned}
$$

\section{La morphologie comme effet (de la phonologie)}

Je fais l'hypothèse que la morphologie est fondamentalement un effet de l'application de la phonologie à des structures de type syntaxique. Cela revient à n'admettre dans la grammaire qu'une seule composante productive, une seule combinatoire (comme l'admet la morphologie distribuée, en particulier Marantz, 1997). Une combinatoire productive d'items lexicaux (morphèmes) est une composante nécessaire de la grammaire dans toute approche qu'on puisse en avoir. Cette combinatoire peut directement être interprétée linéairement si elle est d'un format comme X'où le branchement est à chaque fois au plus binaire [voir Kayne (1994) pour le lien entre linéarisabilité et binarité]. L'interprétation directe d'une structure hiérarchique en successivité relève de la syntaxe (c'est la partie « phonologique » de la syntaxe, la nécessité de la successivité découlant du caractère phonique du langage).

Or, pour l'instant, je me place dans l'hypothèse où la phonologie crée de la linéarité indépendamment de la syntaxe, à l'intérieur des morphèmes. Si le processus de linéarisation proprement phonologique (basiquement la syllabation) 
peut définir aussi l'ordre de réalisation des morphèmes, syntaxe et phonologie comportent des capacités de linéarisation potentiellement concurrentes. Cette concurrence peut éclairer l'existence notoirement problématique du mot dans le langage. Le mot n'est pas une propriété nécessaire du langage, à la différence des morphèmes et des syntagmes, respectivement comme signes élémentaires et comme combinaisons. S'il n'y avait pas de phonologie, il n'y aurait pas de raison que la combinaison des morphèmes relève d'autre chose que des modalités de la syntaxe. Dans une langue à support vocal-auditif, la morphologie et le mot sont l'effet de la possibilité de linéariser phonologiquement des dépendances de type syntaxique.

Pour donner à la phonologie la capacité de créer de la linéarité entre morphèmes et non pas seulement dans les morphèmes, il suffit d'admettre que le processus de linéarisation entamé dans un morphème peut se poursuivre dans un autre. Spécifiquement j'admets le prolongement du processus de morphème à morphème de la manière suivante :

(12) Développement de la linéarisation entre morphèmes :

la linéarisation entreprise dans un morphème tête, une fois le contenu phonique de ce morphème épuisé, peut se poursuivre dans son dépendant immédiat, puis, le processus parvenu au dernier dépendant, dans les adjonctions, en commençant par celles qui sont structuralement les plus proches de ce dernier dépendant (les plus enchâssées).

Soit en (13) un exemple de structure organisant hiérarchiquement des morphèmes (structure mise en place sur la base des relations de sélection entre morphèmes).

$$
\begin{aligned}
& \text { «désenfumage » } \\
& {\left[_{\mathrm{N}} \mathrm{a}-\boldsymbol{z}-\boldsymbol{N}\left[_{\mathrm{V}} \mathrm{d}-\mathrm{e}-\mathrm{z}-\boldsymbol{N} \boldsymbol{e} \boldsymbol{g}\left[_{\mathrm{V}}[\mathrm{a}-\mathrm{N}-\boldsymbol{P L}][\ni-\boldsymbol{V}[\mathrm{f}-\mathrm{y}-\mathrm{m}-\boldsymbol{R}]\right.\right.\right.}
\end{aligned}
$$

La linéarisation d'une telle forme suit les étapes suivantes :

$$
\begin{aligned}
& { }_{\mathrm{N}} \text { a-z- } \boldsymbol{N}^{\circ}\left[_ { \mathrm { V } } \text { d-e-z-Nég } \left[_ { \mathrm { V } } [ \mathrm { a } - \mathrm { N } - \boldsymbol { P L } ] \left[{ }_{3}-\boldsymbol{V}^{\circ}[\mathrm{f}-\mathrm{y}-\mathrm{m}-\boldsymbol{R}]\right.\right.\right. \\
& 1: \mathrm{a}^{\circ}(3) \\
& \begin{array}{lllll}
1 & 5 & 4 & 2 & 3
\end{array} \\
& \text { 2: } \partial^{\circ} \mathrm{a}^{\circ}(\mathrm{z}) \rightarrow \partial^{\circ} \mathrm{a}^{\circ}(\mathrm{z}) \\
& \text { 3: }\left(\mathrm{f} \mathrm{y}^{\circ}\right)\left(\mathrm{m}\left(\mathrm{a}^{\circ} \mathrm{z}\right)\right. \\
& \text { 4: } \mathrm{a}^{\circ}(\mathrm{N})\left(\mathrm{f} \mathrm{y}^{\circ}\right)\left(\mathrm{m}\left(\mathrm{a}^{\circ} \mathrm{z}\right)\right. \\
& 5 \text { : }\left(\mathrm{d} \mathrm{e}^{\circ}\right)\left(\mathrm { z } ( \mathrm { a } ^ { \circ } ( \mathrm { N } ) ) ( \mathrm { f } ^ { \circ } ) \left(\mathrm{m}\left(\mathrm{a}^{\circ} \mathrm{Z}\right)=\right.\right.\text { [dezũfymaz] }
\end{aligned}
$$

Aussi bien qu'un exemple purement dérivationnel, on peut prendre un exemple comportant aussi un morphème flexionnel. Le traitement est le même :

$$
\begin{aligned}
& \text { « Reverdissait » } \\
& {\left[\varepsilon-\boldsymbol{T} / \boldsymbol{A g r}{ }^{\circ}[[\mathrm{r}-\partial-\boldsymbol{A d} \boldsymbol{v}][\mathrm{i}-\mathrm{s}-\boldsymbol{V}[\mathrm{v}-\varepsilon-\mathrm{r}-\mathrm{d}-\boldsymbol{A d j}]]]\right]} \\
& \begin{array}{llll}
1 & 4 & 2 & 3
\end{array}
\end{aligned}
$$


La linéarisation phonologique de structures organisant hiérarchiquement des morphèmes prédit non seulement le « principe du miroir » (Baker, 1985) qui énonce le parallélisme d'organisation hiérarchique entre morphologie et syntaxe, mais le principe de la tête à droite (Williams, 1981) qui enregistre la tendance des têtes morphologiques à occuper la partie droite (la fin) des mots. Les suffixes qui modifient la catégorie sont à droite et le dernier est décisif pour identifier la forme : «nuag(e)-eux » est un adjectif, « grand-it » est un verbe, « a-grand-issement » un nom, etc. Les morphèmes flexionnels correspondant aux têtes fonctionnelles sont encore plus à droite. Si l'approche que j'avance est correcte, cette situation est la conséquence du caractère rétrograde de la linéarisation et devrait valoir universellement. Un préfixe dérivationnel putatif devrait pouvoir en fin de compte s'analyser comme une tête syntaxiquement linéarisée puis cliticisée, ou comme une adjonction à la tête.

Insistons sur le fait que les notions de « suffixe» ou de «préfixe » sont des notions dérivées. Elles ne sont que l'effet du traitement par la phonologie du statut de tête ou d'adjonction structurales. Reste à définir ce qui détermine le traitement affixal, c'est-à-dire phonologique, d'une relation structurale unissant deux morphèmes plutôt que son traitement syntaxique (direct). J'utilise deux propriétés qui déclenchent l'interprétation affixale : la taxicité (ou visibilité) notée $\mathrm{T}$ et la linéarisabilité spontanée notée $\mathrm{L}$. La première détermine la capacité à occuper une place en syntaxe, à être donc un objet pour la linéarisation explicite. Je la suppose appartenir fondamentalement, mais non exclusivement, aux catégories lexicales $(\mathrm{N}, \mathrm{V}, \mathrm{A} . .$.$) . De là, elle se propage vers les têtes supérieures tant que$ celles-ci le permettent (fondamentalement si elles ne sont pas «taxiques » ellesmêmes). La seconde propriété oppose des morphèmes actifs, où le processus de syllabation-linéarisation s'enclenche spontanément et des morphèmes inertes qui ne sont syllabés et linéarisés que par le développement d'un processus issu d'un autre morphème. Je suppose aussi que les radicaux possèdent cette activité, et qu'elle monte vers les têtes qui ne l'ont pas. Si taxicité et activité (autonomie de linéarisation) coïncident, on obtient un domaine de linéarisation homogène, sinon on observe des effets de cycle (plusieurs processus de syllabations enchâssés dans un domaine taxique).

Je ne peux discuter davantage ces notions ici déjà évoquées dans Sauzet (1994a, 1994b). (16) illustre la montée de la taxicité (T) associée à la linéarisabilité (L) du radical « fum- » vers les têtes structurales successives. (17) montre l'opposition entre un préfixe inerte « in- » et un préfixe actif « en- » (Sauzet, 1998a).

16)

$$
\begin{aligned}
& \text { « désenfumage » [ voir (13)] } \\
& { }_{\mathrm{N}} \mathrm{a}-\boldsymbol{z}-\boldsymbol{N o m}{ }_{\mathrm{V}} \mathrm{d}-\mathrm{e}-\mathrm{z}-\boldsymbol{N} \boldsymbol{e} \boldsymbol{g}\left[_{\mathrm{V}}[\mathrm{a}-\mathrm{N}-\boldsymbol{P a r t}][\mathrm{J}-\mathrm{V}[\mathrm{f}-\mathrm{y}-\mathrm{m}-\boldsymbol{R}]\right. \\
& \mathrm{T} / \mathrm{L} \\
& { }_{\mathrm{N}} \mathrm{a}-\mathrm{z} \text {-Nom }{ }_{\mathrm{V}} \mathrm{d} \text {-e-z-Nég }\left[_{\mathrm{V}} \text { [a-N-Part }\right][\mathrm{\jmath}-\mathrm{V}[\mathrm{f}-\mathrm{y}-\mathrm{m}-\boldsymbol{R}] \\
& \mathrm{T} / \mathrm{L} \quad \leftarrow \quad(\mathrm{T} / \mathrm{L}) \leftarrow(\mathrm{T} / \mathrm{L})
\end{aligned}
$$


C'est la tête «-age » qui est finalement taxique (le nom « désenfumage » est un objet de placement syntaxique) et où se déclenche la linéarisation par syllabation.

$$
\text { a. } \begin{array}{r}
\mathrm{C}_{\mathrm{Adj}}[\mathrm{i}-\mathrm{N}-\boldsymbol{n e ́} \boldsymbol{g}] \mathrm{C}_{\mathrm{Adj}} \text { a-b-l-Adj }\left[_{\mathrm{V}} \mathrm{\jmath}-\boldsymbol{V}[\mathrm{u}-\mathrm{b}-\mathrm{l}-\mathrm{i}-\boldsymbol{R} \boldsymbol{a d}]\right. \\
\mathrm{T} / \mathrm{L} \leftarrow(\mathrm{T} / \mathrm{L}) \leftarrow(\mathrm{T} / \mathrm{L})
\end{array}
$$

un seul domaine de syllabation : [inubliabl]

b. $\left[_{\mathrm{V}}[\mathrm{a}-\mathrm{N}-\boldsymbol{P a r t}]\left[_{\mathrm{V}}\right.\right.$ ə-V $\left[_{\mathrm{Adj}}\right.$ i-v-r-Adj $]$

$$
\mathrm{L} \quad \mathrm{T} / \mathrm{L} \stackrel{\mathrm{Adj}}{\leftarrow}(\mathrm{T} / \mathrm{L})
$$

deux domaines de syllabation : [[đ] (n)ivr] (l'un enchâssé dans l'autre, $\mathrm{d}$ 'où la resyllabation donnant $[\mathrm{n}]$ antihiatique après voyelle nasale).

L'analyse proposée considère donc qu'une forme suffixée comme [ $\left.\int t \varepsilon\right]$, « chantait » est la réalisation in situ (par construction phonologique) de la configuration structurale [ $\varepsilon$-imparf. [ [-a-N-t-Verbe $]]$. On peut envisager d'étendre cette conception aux faits de morphologie non concaténative. Le point crucial de cette extension consiste à admettre qu'au lieu que la syllabation traite successivement un ensemble tête-dépendant (à partir de la tête), elle traite conjointement leur contenu phonique. Supposons que certaines têtes déclenchent ce processus que je continuerai à appeler « fusion » (malgré les usages divers de ce mot) : les propriétés non phoniques sont intégrées et identifient globalement l'objet phonologique construit. Par fusion on lit donc (18a) comme (18b).
a. $\left[\mathrm{a}-\right.$ prétérit $^{\circ} \quad[\mathrm{r}-\Lambda-\mathrm{n}-\text { Verbe }]^{15}$
b. a-prétérit ${ }^{\circ}$ Verbe $-\mathrm{n}-\Delta-\mathrm{r}$

Ce qui est remarquable en (18 b), c'est la présence d'un matériau phonique discontinu. Supposons que le processus de syllabation s'enclenche dans chaque fragment phonologique. On passe dès lors par la phase suivante, (19), où les deux processus enclenchés sont notés sur les lignes a) et b) :
a.
$\mathrm{a}^{\circ}$ Prétérit
b. $\mathrm{r}-\mathrm{\Lambda}^{\circ}-(\mathrm{n})$ Verbe

Si la ligne b de (19) était seule en cause, l'étape suivante verrait la formation d'une relation attaque-noyau entre $/ \mathrm{r} /$ et $/ \mathbb{N}$. Si la voyelle caractéristique du prétérit, /a/, avait été une tête non fusionnante (suffixale), elle aurait fait de /n/, segment le plus accessible du radical, une attaque. Supposons que la fusion rende les segments les plus accessibles (ici /a/ et /n/) mutuellement inaccessibles. En revanche /r/, si l'attenance n'est pas une condition à la mise en relation d'un noyau et d'une attaque, peut aussi bien être l'attaque d'une syllabe ayant $\Lambda^{\circ}$ pour noyau que $\mathrm{a}^{\circ}{ }^{16}$. On peut représenter le résultat comme suit, où le noyau syllabique est ambigu :

$$
\left((\mathrm{r})\left(\Lambda^{\circ} / \mathrm{a}^{\circ}(\mathrm{n})\right)\right.
$$


La structure en (20) ne peut évidemment être réalisée comme telle et il faut choisir une voyelle unique dans la réalisation effective. Si la mobilisation de /a/ conditionne celle du trait non phonique de prétérit auquel il est attenant, c'est cette voyelle qui sera systématiquement mobilisée au prétérit, au détriment de $/ N$ non marqué pour le temps (éventuellement marqué pour le présent).

Cette rapide incursion dans la morphologie non concaténative a deux objectifs. D'une part suggérer que la « syntaxe des mots », celle qui agence les morphèmes, ne doit pas nécessairement être directement lisible dans l'agencement linéaire de la réalisation phonique, comme la syntaxe des phrases. McCarthy $(1982$ : 222) et à sa suite Spencer $(1991: 147)$ relèvent qu'on ne peut dans une morphologie non concaténative repérer par un parenthésage une structure de constituants. En fait, on ne doit s'attendre à ce que ce soit possible que dans la mesure où la phonologie traite successivement les morphèmes. La disposition linéaire des spécifications phoniques résulte de l'interaction de la construction des constituants phonologiques avec l'organisation hiérarchique initiale des morphèmes. Cette réfraction par la phonologie a pour effet la finalité de la tête quand la morphologie est concaténative (successive), l'interdigitation quand la morphologie est non concaténative (simultanée ou "fusionnelle") ${ }^{17}$.

D'autre part, en relation plus immédiate avec la question des consonnes latentes, il s'agissait de montrer que l'approche ici défendue peut utiliser des représentations où le contenu phonique est discontinu, comme l'objet dérivé en (19). Le processus de syllabation permet d'intégrer le matériau discontinu dans les constituants phonologiques finalement construits et réalisés. Je me propose de traiter les consonnes latentes du français comme un cas de présence lexicale d'un contenu phonologique discontinu, ce que le format des morphèmes retenu ici rend parfaitement possible.

\section{Latence}

Quel statut donner aux consonnes latentes typiques de la phonologie du français et qui se manifestent dans la liaison, la réalisation du féminin et la dérivation? Rappelons en (21) un paradigme typique :

$$
\text { «petit » [pəti], «petit ami » [pətitami], «petite» [pətit], «petitesse » [pətites] }
$$

Les caractérisations des consonnes latentes qui ont été proposées en font soit des morphèmes, soit des segments ordinaires, soit des segments déficients.

L'analyse de Marguerite Durand, centrée sur le genre, considère ces segments comme des marques de féminin et en ce sens comme des morphèmes (Durand, 1936) ${ }^{18}$. Cela suppose bien sûr de nombreuses classes morphologiques pour le genre en français, mais l'objection principale à cette analyse reste que la présence d'une même consonne au féminin, en liaison et dans la dérivation conduit à considérer qu'elle fait partie de la caractérisation lexicale de la racine (Dell, 1973 : 180). De là l'analyse générative classique (Schane, 1968 ; Dell, 
1973) qui considère les consonnes latentes comme des segments ordinaires soumis en finale absolue (et sauf liaison) à un processus phonologique d'effacement.

Le problème de l'approche générative est l'opacité de la règle d'effacement qu'elle suppose. Pour rendre compte, non seulement de «petit, petite », « lourd, lourde », « gros, grosse », mais aussi de « gentil, gentille », « premier, première », etc., il faut une règle qui efface tous les segments consonantiques finaux, des semi-voyelles aux occlusives sourdes ${ }^{19}$. Dell (1973) pose que toutes les formes immunes d'effacement ont schwa final. Cette solution est acceptable en français standard, mais elle ne peut rendre compte des variétés (méridionales ${ }^{20}$ ) du français qui ont une opposition à trois termes :

$$
\begin{aligned}
& \text { - «petit, petite» [pəti] [pətitə] } \\
& \text { - «net, nette » [nct] [nctə] } \\
& \text { - « honnête, honnête » [onєtə] [onєtə] }
\end{aligned}
$$

Dans les parlers qui ont le paradigme donné en (22) si le rôle préservateur de schwa est transparent (puisque schwa est réalisé), il est tout aussi manifeste qu'il n'explique pas toute la conservation.

Ce n'est toutefois pas à cause des formes du type de (22) mais plutôt parce que le recours systématique à schwa dans les formes non alternantes semble une solution trop abstraite et parce que le renouvellement du formalisme phonologique offre des solutions nouvelles, que diverses analyses en termes de segments déficients ont été avancées, à partir de Clements \& Keyser (1981). Voir Encrevé (1988a, 1988b) pour un traitement classique de ce type et une récapitulation exhaustive. De telles analyses exploitent les possibilités ouvertes par les représentations multilinéaires de maintenir un traitement phonologique en échappant à l'opacité de la règle d'effacement des consonnes finales. Pierre Encrevé relève que la notion de segment flottant est venue donner un statut phonologique à la supplétion lexicale proposée comme alternative au traitement génératif classique (Encrevé, 88b : 87-96) ${ }^{21}$. Les analyses multilinéaires de la latence distinguent la position et le contenu : les consonnes latentes, dites de ce fait « flottantes », ont le même contenu que des segments ordinaires, mais ne sont pas associées à une position qui en assure la réalisation constante. Outre que le flottement est une interprétation fondée de la déficience segmentale, il permet de traiter les dialectes à trois classes, comme on voit en (23).

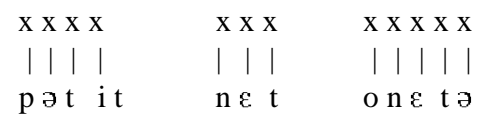

La faiblesse principale de l'approche multilinéaire, c'est qu'elle doit poser que le segment flottant est marqué par l'absence d'une propriété qui est redondante dans les autres segments. Si le seul encodage de la linéarité est une suite de positions 
(notée par une concaténation d'objets identiques : x x x...), deux problèmes se posent : d'une part c'est un encodage implicitement numérique, d'autre part le segment flottant, n'étant pas ancré, n'est nulle part (ni final, ni initial, ni avant, ni après un autre puisque, par hypothèse, seules les positions encodent la successivité), sauf par illusion typographique. Si par contre la suite de positions (notée : x x x...) n'est pas le seul encodage de la successivité, cela signifie que les contenus segmentaux sont linéarisés par eux-mêmes (indépendamment de leur relation à la ligne des positions). Cela peut s'écrire :

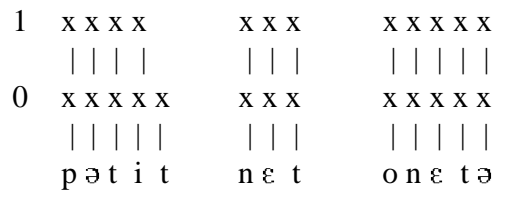

Ci-dessus les $\mathrm{x}$ de la ligne 0 marquent la successivité (par hypothèse les segments notés au-dessous n'ont pas d'ordre indépendamment de leur attachement à cette ligne). La ligne 1 sert à distinguer segment flottant et segments ordinaires, et elle le fait en supposant aux segments ordinaires une position de plus. Cette figure (24) fait clairement apparaître qu'il faut, dans un cadre autosegmental, enrichir les segments ordinaires pour représenter la déficience des segments flottants.

Un acquis de l'analyse générative classique est que les consonnes latentes sont lexicales : c'est une propriété du radical «petit », réalisé [pəti] en isolation que de présenter un [-t-] au féminin, en dérivation et en liaison. De même c'est une propriété des suffixes «-ot, -ote » et «-aud, -aude » de présenter des consonnes latentes différentes.

L'approche multilinéaire apporte l'idée que ces segments ont un statut particulier, une déficience qui les prédispose à l'effacement. Cette déficience ne saurait concerner la caractérisation phonique (traits ou éléments) : tout segment peut être latent. Sauf à poser un diacritique $a d h o c$, la seule autre information que le contenu phonique d'un segment est celle qui détermine sa relation aux autres éléments, phoniques ou non, du morphème où il figure. Si l'information phonique est une concaténation de segments, le segment flottant ne peut être exclu de cette concaténation sauf à être aussi exclu du morphème, d'où le redoublement de la concaténation auquel doit procéder une approche autosegmentale pour noter la déficience, voir (24).

Supposons que les segments latents ont pour particularité d'appartenir à un morphème mais de ne pas être attenants au reste de l'information phonique d'un morphème. Le contenu phonique des morphèmes qui comportent un segment flottant est discontinu. C'est donc un objet du même type de celui qui résulte de la fusion de deux morphèmes [voir (19) ci-dessus] ${ }^{22}$.

On opposera donc :
a. $\quad \mathrm{p}-\partial-\mathrm{t}-\mathrm{i}-\boldsymbol{A} d \boldsymbol{d}$-t
b. $\mathrm{n}-\varepsilon-\mathrm{t}-\boldsymbol{A} d \boldsymbol{d j}$
c. o-n- $\varepsilon-\mathrm{t}-\partial-A d j$ 
En français standard, l'opposition de (25b) et (25c) est neutralisée au profit, peuton poser, de la forme la plus pauvre, soit (25b).

En (25a) la caractérisation phonique du morphème n'est pas globalement connexe mais se répartit en deux fragments. Le segment latent se caractérise par sa dissociation du reste du contenu phonique. La dissociation, comme le flottement, est en un sens une réinterprétation de la supplétion. Elle a l'avantage tout d'abord de ne pas inverser la marque comme l'analyse autosegmentale et ensuite de permettre, comme on le verra, le traitement de cas moins transparents que celui de la consonne latente constante d'une forme comme «petit».

En isolation, chaque fragment de contenu phonique d'une forme comme (25a) est linéarisé indépendamment. Soit :
a. [pəti]
b. $[\mathrm{t}]$

Chacun des objets ainsi obtenu, (26a) et (26b), est candidat à réaliser le morphème. J'utilise à dessein le terme "candidat" emprunté à la théorie de l'optimalité [pour cette approche, je me contente ici de renvoyer à McCarthy \& Prince (1997) et aux références qui s'y trouvent]. Je me tiens néanmoins dans une conception dérivationnelle de la phonologie : il y a dérivation en particulier parce que la linéarité est nécessaire à la réalisation, mais pas la caractérisation lexicale. Idéalement la dérivation ne comporte pas de stipulations spécifiques mais seulement l'instruction « linéariser » qui se traduit par la syllabation, la construction de structure métrique et leurs effets... L'évaluation ne s'impose que quand le processus dérivationnel est susceptible de plusieurs issues. Il s'agit donc, si l'on tient à parler d'optimalité, d'une optimalité restreinte ${ }^{23}$. Si en (26) le meilleur candidat doit être retenu, (26a) s'impose, tant par la fidélité aux spécifications lexicales que par sa viabilité phonologique.

Que se passe-t-il en cas de suffixation ? Typiquement dans une langue romane, les suffixes sont à initiale vocalique. Cette initiale est identifiée comme tête et le processus se poursuit, incluant le radical. Ici, plusieurs segments sont également accessibles : /i/ et /t/ pour « petit», en (26a). La consonne dissociée, que l'algorithme de syllabation identifie comme une syllabe dégénérée, permet de former une attaque au contraire de la tête $\mathrm{i}^{\circ}$. J'admets donc que cette résolution « optimale » est choisie. Notons que le processus de syllabation issu de la tête suffixale peut incorporer, d'une part la consonne dissociée, d'autre part le reste du contenu phonique du morphème. La, ou les syllabes qui réalisent la masse du morphème, sont adjointes à gauche du domaine de syllabation déjà ouvert par le suffixe.

Il faut remarquer l'effet variable de la dissociation. Quand le processus de linéarisation commence avec le morphème contenant un segment dissocié, deux processus indépendants se développent et le «meilleur » résultat (le plus fidèle, le plus prononçable...) est seul réalisé. Quand le processus commence dans une tête supérieure, il est capable d'intégrer la consonne dissociée comme la partie la plus substantielle du radical. C'est aussi bien le cas quand la tête flexionnelle n'est 
que schwa : cette voyelle minimale (indispensable pour représenter un effet vocalique sans voyelle réalisée) mobilise la consonne dissociée et forme avec elle une syllabe. À cette syllabe s'adjoignent celles qui se forment à partir du reste du contenu phonique du radical, permettant alors que la syllabe finale comportant schwa soit intégrée dans un pied.

La dissociation (qu'autorise une conception non prélinéarisée des morphèmes) constitue un moyen de saisir la déficience propre aux consonnes latentes. Celles-ci sont des fragments de spécification des morphèmes, ce qui prend en compte leur caractère lexical reconnu par la phonologie générative classique. La réinterprétation de leur déficience en dissociation exprime toutefois leur spécificité en leur donnant un statut de «quasi-morphèmes », en ce que ce sont des parties phoniques connexes (trivialement) de morphème. L'effet d'un morphème ne consistant qu'en schwa est de mobiliser ces quasi-morphèmes au féminin.

Les alternances que présente un adjectif à consonne finale latente correspondent aux représentations et résolutions suivantes :

a. forme isolée

LEX : $\quad$ p-כ-t-i-Adj-t

Résolution $[(\mathrm{p} \ni)(\mathrm{ti})] / *[(\mathrm{t})]$

b. dérivation

LEX : $\quad$ p-כ-t-i-Adj-t; $\varepsilon-\mathrm{s}-\mathrm{Nom}$

SINT : [e-s-Nom [p-כ-t-i-Adj-t]]

Résolution : [(pə)(ti)(tes)]

c. féminin

LEX : $\quad$ p-ə-t-i-Adj-t; כ-Fém

SINT : [ə-Fém [p-כ-t-i-Adj-t]]

Résolution : $\quad[(\mathrm{p} \ni)\{(\mathrm{ti})(\mathrm{t})\}]$

L'objet noté entre \{ \} est un pied, ou une super-syllabe comme on voudra, en tous cas un objet formé d'une syllabe dont le noyau est une voyelle forte et d'une syllabe dont le noyau est schwa. La syllabe finale contenant un schwa est d'abord, comme dans les autres cas, celle où s'adjoignent les autres syllabes formées ensuite. La relation d'adjonction est inversée en gouvernement si une syllabe pleine doit être adjointe à une syllabe ayant schwa pour tête :

$$
\ldots \ldots\left(_{\partial}(\mathrm{ti})(\mathrm{t} \ni)\right) \rightarrow \quad \ldots .\left(_{\mathrm{i}}(\mathrm{ti})(\mathrm{t} \partial)\right)
$$

La liaison obligatoire concerne en général des éléments clitiques précédant le nom ou le verbe que l'on peut supposer intégrés dans le domaine de syllabation de ces catégories. Les adjectifs prénominaux sont les seules formes lourdes déterminant la liaison obligatoire ou « invariable » (Encrevé, 1988a : 47, voir aussi Abeillé et Godard ici-même). Je pose que l'absence de taxicité (Sauzet, 1998b) fait que ces 
adjectifs ne peuvent recevoir une place linéaire que par leur intégration au domaine de syllabation du nom dont ils dépendent :
a. [y-n-dét. []-a-p-o-Nom-t [b-1-ø-Adj ...
$\mathrm{T} \quad \mathrm{T}$
b. [y-n-dét. [f-a-p-o-Nom-t [3-J-1-i-Adj...
$\mathrm{T}$

En (29a) « chapeau » et « bleu » sont tous deux taxiques (syntaxiquement visibles). Les deux objets phonologiques (domaines de syllabation) qu'ils constituent peuvent donc être ordonnés l'un par rapport à l'autre par interprétation directe en successivité de la structure hiérarchique. En (29b) « joli » n'est pas taxique. Il ne peut se trouver réalisé qu'en tant qu'il est associé à un domaine taxique, celui de «chapeau ». Une fois «chapeau » réalisé, les syllabes de «joli » qui est structuralement sœur de ce domaine sont adjointes à gauche comme le sera en suite celle de l'article indéfini. Autrement dit le matériau phonique des morphèmes (ou conjonction de morphèmes) non taxiques structuralement liés à un domaine taxique est traité comme celui de morphèmes adjoints, linéarisés dans l'ordre de leur proximité structurale avec le morphème taxique.

Si l'adjectif non taxique comporte une consonne dissociée (latente) celleci peut être intégrée comme attaque dans le processus de syllabation ${ }^{24}$ issu d'un nom à initiale vocalique, avant que le reste du contenu phonique de l'adjectif soit adjoint à gauche. Soit donc :

a. Liaison

LEX : $\quad$ a-m-i-Nom p-ə-t-i-Adj-t

SINT : $\quad$... [ a-m-i-Nom $[\mathrm{p}-\partial-\mathrm{t}-\mathrm{i}-\boldsymbol{A}$ Adj-t $]] \ldots$

$\mathrm{T}$

b. Résolution :

i) $\left.\quad \ldots \mathrm{a}^{\circ}-\left(\mathrm{mi}^{\circ}\right)\right] \quad$ syllabation de « ami »

ii) $\left.\quad \ldots\left(\mathrm{ta}^{\circ}\right)\left(\mathrm{mi}^{\circ}\right)\right]$ accès au matériau de « petit »

iii) $\quad\left[(\mathrm{p} ə)(\mathrm{ti})\left(\mathrm{ta}^{\circ}\right)\left(\mathrm{mi}^{\circ}\right)\right] \quad$ épuisement du matériau de « petit »

$\mathrm{Si}$ /t/ dissocié n'est pas intégré à la syllabe initiale du nom (taxique), le processus de syllabation doit construire une syllabe nouvelle avec le matériau de l'adjectif. Comme dans le cas de l'emploi isolé, deux processus parallèles s'enclenchent et seul celui dont le résultat est optimalement prononçable prévaut (d'où « petit copain $\left.\gg\left[(\mathrm{p} ə)(\mathrm{ti})\left(\mathrm{ko}^{\circ}\right)(\mathrm{p} \widetilde{\varepsilon})^{\circ}\right)\right]$ et non $\left.*\left[(\mathrm{t})\left(\mathrm{ko}^{\circ}\right)(\mathrm{p} \widetilde{\varepsilon})^{\circ}\right)\right]$

Ci-dessus j'ai supposé que « petit » n'était pas syllabé quand le processus issu du nom l'atteint. J'ai proposé ailleurs que pour les éléments ainsi intégrés dans un processus de syllabation qui leur assigne une place linéaire, il peut y avoir soit syllabation primaire (si ce qui est intégré est un morphème inerte qui n'est pas syllabé encore) soit resyllabation (si c'est un morphème " actif » qui a déjà été syllabé, ayant la propriété L). [Voir 2 ci-dessus, en particulier (17) et le paragraphe qui précède (16).] 
On opposera ainsi en liaison «bon », « divin » non-taxiques (quand ils sont prénominaux) et inertes et « un », « mon », « malin », « commun » non taxiques mais actifs. Comparons les traitements de «divin» et de «malin» devant voyelle ${ }^{25}$ :

(31) Liaison d'adjectif inerte à finale nasale : « divin Ulysse »

LEX : $\quad \mathrm{y}-\mathrm{l}-\mathrm{i}-\mathrm{s}-\operatorname{Nom}(\mathrm{T}, \mathrm{L})$; d-i-v-i-N-Adj-t

SINT : ... [y-l-i-s-Nom [d-i-v-i-N-Adj-t]]...

$\mathrm{T}, \mathrm{L}$

résolution :

i) $\quad \ldots y$ - (lis)] syllabation du nom taxique

ii) $\quad[(\mathrm{di})(\mathrm{vi})(\mathrm{ny})(\mathrm{lis})] \quad$ accès au matériau non taxique associé

(32) Liaison d'adjectif actif à finale nasale : « malin esprit»

LEX : e-s-p-r-i-Nom-t (T, L) ; m-a-l-i-N-Adj-t (L)

SINT : ... [e-s-p-r-i-Nom [m-a-l-i-N-Adj-t]]...

$\mathrm{T}, \mathrm{L}$

résolution :

i) $\quad \ldots \varepsilon^{\circ}$-(s)- (pri)] syllabation du nom taxique

(ma) (liN)] syllabation parallèle de l'adj. non taxique

(mal) (le)) résolution de $\mathrm{i}^{\circ} \mathrm{N}$ en $\left.[\varepsilon)\right]$

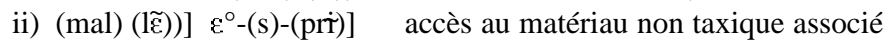

(déjà syllabé)

$\left.[(\mathrm{mal})(\mathrm{l} \widetilde{\varepsilon}))\left(\mathrm{n} \varepsilon^{\circ} \mathrm{s}\right)-(\mathrm{pri})\right] \quad$ resyllabation et développement d'une attaque

nasale épenthétique

Les adjectifs qui déclenchent une liaison où se manifeste une consonne latente sont non taxiques, d'où leur position prénominale. Admettons aussi qu'ils sont inertes (que ne s'y déclenche pas de processus de syllabation autonome). La dérivation donnée ci-dessus en (30) peut donc être maintenue. Si la réalisation du segment dissocié suppose l'inertie du morphème auquel il appartient, cela suggère qu'il peut exister des adjectifs prénominaux dont la consonne dissociée (latente) n'est pas activée en liaison. C'est le cas dans l'usage ordinaire pour un adjectif comme « fort » illustré en (33b) et (33c) :
a. (for)(ta)(ksã))
f-כ-r-Adj.-t ni T ni L(non taxique inerte)
b. $(\mathrm{for})(\mathrm{a})(\mathrm{ks} \widetilde{\mathrm{a}}))$
f-כ-r-Adj.-t L (non taxique, actif)
c. $(\mathrm{f} \ni)(\mathrm{ra})(\mathrm{ks} \tilde{a}))$
L et resyllabation

On expliquera aussi l'observation d'Yves-Charles Morin que les adjectifs employés en position prénominale forcée sont volontiers laissés sans consonne de liaison : « un [frã] entretien » (Morin, 1992 : 242) : il est sans doute difficile de rendre un morphème inerte quand il a par ailleurs des emplois actifs. Ce qui est en jeu ce n'est pas la consonne latente comme le pose Morin : ses témoins n'auraient sans doute pas hésité à dériver sur un radical [bla]-] des mots 
apparentés à «blanc» («blanchoyer » ou «blancherie», etc.). S'ils ne mobilisent pas [ [] en liaison, c'est qu'il faudrait acquérir l'inertie de «blanc » qui le fasse intégrer non syllabé au domaine de syllabation du nom suivant ${ }^{26}$.

Un intérêt de l'approche qui voit dans les consonnes latentes des contenus phoniques dissociés (du reste du contenu phonique) est qu'elle permet d'envisager des situations plus variées que la seule latence canonique de «petit, petit-t-ami, petitesse ».

Envisageons d'abord les cas où la consonne de liaison diffère de la consonne de dérivation, comme « grand, grand-t-ami, grandeur » « gros, gros-zami, grosseur » « frais, frais-z-ombrage, fraîche, fraîcheur ». On posera à la base de ces formes un morphème du format suivant (avec deux segments dissociés).

$$
\begin{gathered}
\text { g-r-a-N-Adj }-\mathrm{d} \\
\mathrm{t}-\text { masc }
\end{gathered}
$$

En supposant 1 un trait de genre «masculin »spécifiquement lié au contenu phonique dissocié /t/, on peut admettre que la mobilisation de /t/ entraîne celle de ce trait. Les deux consonnes dissociées ${ }^{27} / \mathrm{d} /$ et /t/ sont en concurrence pour être attaque d'une voyelle suffixale ou initiale du nom suivant. Dans un contexte où la mobilisation du trait masculin est souhaitable, celle de /t/ sera favorisée (liaison) et celle de /d/ dans le cas inverse (dérivation) ${ }^{28}$. Notons bien que /t/ étant dissocié n'est pas réalisé, malgré son lien à un trait d'accord, si la forme n'est pas en contexte de liaison : la présence du trait masculin ne permet que de privilégier /t/ dans des contextes où une consonne dissociée peut être réalisée, elle ne peut imposer cette réalisation) :

$$
\begin{gathered}
{[\mathrm{t}-\operatorname{masc} \text { [g-r-a-N-Adj-d } \rightarrow \quad \mathrm{g}-\mathrm{r}-\mathrm{a}-\mathrm{N}-\boldsymbol{A d j}-\mathrm{d}} \\
/ \\
\mathrm{t} \longrightarrow \text { masc }
\end{gathered}
$$

On peut, suivant Morin (1992), admettre que t-masc est un morphème masculin (de même z-masc pour « gros »). Si c'est le cas cela expliquerait la tendance de certains locuteurs à généraliser /t/ de liaison : « fran-t-ananas » notée par Morin (1992: 242-243) dans le cas d'antéposition forcée de l'adjectif (qu'il a fait réaliser à ses informateurs) et dont il rappelle qu'elle représente une liaison fautive classique. Supposons ce / $t$ / inerte et fusionnant, supposons aussi qu'il reste inerte après fusion (il n'est pas mobilisable par le processus de syllabation à l'œuvre dans l'adjectif). Seul le processus de liaison peut mobiliser /t/, qui sera à ce titre un instrument passe-partout de la fausse liaison.

Le morphème de pluriel z-plur est un morphème fusionnant de ce type. Supposons qu'une tête pluriel est présente au-dessus de chaque morphème pluralisable d'un groupe nominal (déterminant, adjectifs, nom lui-même). 


$$
\text { [z-plur [1-e-Dét.... [z-plur [a-m-i-Nom }{ }^{29}
$$

La fusion donne :

$$
\begin{array}{cc}
\text { /1-e-Dét ... } & {[\mathrm{a}-\mathrm{m}-\mathrm{i}-\mathrm{Nom}} \\
\text { z-plur } & \text { z-plur }
\end{array}
$$

Si l'on suppose encore que, quoi qu'il en soit de l'inertie du déterminant, le morphème de pluriel reste inerte, le processus de linéarisation issu de «ami » donne successivement :

$$
\begin{aligned}
\left.\ldots \mathrm{a}^{\circ}(\mathrm{mi})\right] & \text { (et parallèlement }[\mathrm{z}] \text { concurrent et non retenu) })^{30} \\
\ldots \mathrm{za})(\mathrm{mi})] & \text { mobilisation du segment dissocié du morphème accessible } \\
\text { (le) }(\mathrm{za})(\mathrm{mi})] & \begin{array}{l}
\text { épuisement du matériau accessible et intégration au } \\
\text { domaine de syllabation }
\end{array}
\end{aligned}
$$

Le format lexical des morphèmes ici assumé permet de représenter encore des cas d'alternance parallèle à la mobilisation de consonnes latentes mais différents, du type « beau, bel », « mou, mol », « vieux, vieil ». On posera par exemple :

$$
\begin{gathered}
\text { b-o- } \boldsymbol{A d j} \\
/ \\
\varepsilon-1
\end{gathered}
$$

En cas de dérivation ou de liaison obligatoire (variante inerte de ce morphème) la syllabation qui mobilise la consonne accessible (/l/) donne une syllabation moins marquée (avec attaque) et se trouve privilégiée à ce titre. La configuration (39) est interprétée [bel...] dans ces cas là. Une fois /l/ syllabé comme attaque par le suffixe, $\varepsilon^{\circ}$ et $\mathrm{o}^{\circ}$ sont deux candidats possibles à faire de $/ \mathrm{b} /$ leur attaque (l'attenance n'est pas requise pour ce faire). On dérive donc comme en (40) :

$$
\begin{aligned}
& \text { [A-t-r-Nom [b-o-Adj } \\
& \\
& \\
& \text { i) } \\
& \varepsilon-1 \\
& \text { ii) } \quad \ldots \quad \mathrm{a}^{\circ}-(\operatorname{tr}) \\
& \text { iii) } \quad\left[\left(\mathrm{b} \varepsilon^{\circ}\right)(\mathrm{la})(\operatorname{lt})\right]
\end{aligned}
$$

Par contre on pourra poser ${ }^{31}$ que, des deux processus de syllabation qui s'enclenchent quand la forme est isolée, celui qui identifie le plus rapidement une tête syllabique est privilégié (donc celui qui fait de /o/ accessible une tête de syllabe) : 


$$
\begin{gathered}
\mathrm{b}-\mathrm{o}^{\circ}-A d j \\
/ \\
\varepsilon^{\circ}-1 \\
{\left[\left(\mathrm{bo}^{\circ}\right)\right] *[(\mathrm{bcl})]}
\end{gathered}
$$

La syllabation qui identifie immédiatement une tête est aussi privilégiée si le suffixe commence par une consonne d'où : $*$ (bo)(te) $] *[($ bel $)($ te $)]$

La sélection « mou, mol », « fou, fol », « vieux, vieil », « nouveau, nouvel » obéit aux mêmes principes. Notons cependant le fait curieux qu'alors que « mol » et «molle » peuvent être prénominaux, « mou » ne peut l'être, même devant une consonne : « mol oreiller $v s *_{«}$ mou traversin ». On peut supposer que le caractère taxique est, dans cette forme, associé de manière constante à un seul fragment de caractérisation phonique (celle qui est propre à la forme «fou »).

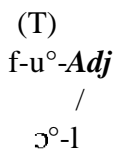

Ainsi, la liaison, ou la voyelle féminine, sélectionne et active le fragment phonique à consonne finale (non taxique) et on obtient [fəl...] prénominal. Si l'allophone contenant la voyelle /u/ est activé, celui-ci est taxique et ne peut être prénominal (marginalement on trouve l'activation de « fol » «ol » devant consonne : « mol bercement » chez Genevoix selon Grevisse). Pourquoi alors « mollesse » est-il taxique ( $\mathrm{si}$ « molle » ne l'est pas) : c'est que la non-taxicité de « mol- » n'est qu'optionnelle d'où « montre molle », et qu'on dérive sur la variante taxique. Au masculin «mou » taxique, et donc post-nominal, est préféré comme « beau » ci-dessus.

Hors des faits de liaison il existe des faits de variabilité de la finale de divers morphèmes qui dans la présente approche relèvent du même type de représentations que la latence, régulière («petit») ou complexe (« grand »). Il s'agit de formes présentant une consonne réalisée différente au masculin d'une part, au féminin et en dérivation de l'autre : «sec [sek], sèche », « craintif, -ive, -ivité » et tous les mots en «-if, -ve », « chanteur, -euse » et tous les mots en «-eur, -euse », etc. ${ }^{32}$.

On posera des formats de morphèmes du type (42) «-if, -ive » et (42b) (« -eur, -euse ») :

$$
\begin{gathered}
\text { a. } \mathrm{v}-\boldsymbol{A d j} \\
/ \quad / \quad । \\
\mathrm{i}-\mathrm{f}-\operatorname{masc}
\end{gathered}
$$

Ces morphèmes autorisent plusieurs interprétations linéaires et, comme on l'a déjà posé plus haut, l'interprétation mobilisant un trait d'accord est privilégiée ou 
évitée selon que ce trait est congruent ou non au contexte d'emploi de la forme. La différence entre les formes (43) et une forme comme « grand », (35), est que l'ambiguïté de réalisation ne concerne pas des segments dissociés, mais des fragments d'une caractérisation phonique connexe unique.

\section{Conclusion}

Admettre que la linéarité est dérivée permet de diversifier le format lexical des morphèmes et de représenter les consonnes latentes comme des segments dissociés (du reste de la caractérisation phonique). Elle permet aussi d'enregistrer des alternances qui échappent aussi bien au modèle génératif classique que (pour certaines d'entre elles) aux représentations multilinéaires, qu'il s'agisse de latence variable («grand-t-ami », «grande») ou d'alternances entre segments non latents, consonantiques («sec, sèche ») ou vocaliques ${ }^{33}$.

En se donnant des morphèmes de ce format, on peut envisager de se dispenser d'une composante morphologique, même « distribuée » à travers la grammaire. L'effet morphologique résulte de l'interprétation phonologique de configurations syntaxiques. J'ai de plus proposé d'admettre que l'interprétation de ces configurations se fait largement in situ. Dans les morphologies concaténatives la réalisation suffixale d'une tête syntaxique est un effet direct de l'interprétation phonologique de la relation tête-dépendant et ne suppose aucun déplacement syntaxique. Il en va de même pour les morphologies non concaténatives si l'approche en termes de fusion et de cosyllabation esquissée ici et dans (Sauzet, 1994b) peut être maintenue.

Si l'on accepte le maintien in situ d'éléments que l'on suppose volontiers déplacés (verbes par rapports à la tête temporelle et personnelle par exemple), il reste à explorer les effets de l'interprétation phonologique (concaténative ou non) d'une relation tête-dépendant sur les autres éléments présents dans la structure. On peut envisager, en faisant varier les conditions de la résolution phonologique (en termes de présence possible d'éléments adjoints intervenants, de visibilité locale des termes d'un complexe phonologiquement résolu) d'en déduire les effets sur la typologie de l'ordre des constituants phrastiques que l'on attribue à des déplacements destinés à satisfaire des traits dans le cadre minimaliste. Comme dans ce cadre, la résolution morphologique apparaît comme le lieu central de la variation linguistique. Ce qui est proposé ici est de rendre la dépendance plus directe : au lieu qu'un mouvement syntaxique déclenché par un trait rende possible (par adjacence) la résolution morphologique, c'est l'interprétation phonologique d'une structure qui produit des effets directs d'affixation (envisagé ici) ou de cliticisation (Sauzet, 1996), et éventuellement des effets indirects qui restent à explorer.

On peut ainsi esquisser une image globale de la variation linguistique dans laquelle un effet morphologique (résolution en mots) variable résulte du rôle respectif dans une langue des deux sources de la linéarité (syntaxe et phonologie). 
De cet effet variable découlent des ajustements syntaxiques nécessaires tendant à rendre possible la résolution phonologique là où elle est attendue.

\section{NOTES}

1. Que l'on trouvera exposées dans Sauzet (1993, 1994, 1996). Je ne renvoie à ces travaux que sur des points spécifiques, pas pour l'approche globale que je maintiens ici.

2. Voir Spencer (1991) pour une présentation de divers modèles théoriques en morphologie.

3. «La linéarité est un fait de parole» notent très justement Lafont Gardès-Madray (1976).

4. Voir la parabole des œufs écrasés de Charles Hockett évoquée dans la présentation de ce numéro de Recherches Linguistiques de Vincennes (Hockett, 1955 : 210), voir aussi à ce sujet Segui (92:135), Cao ( $85: 272)$.

5. C'est à juste titre que Cao Xuan Hao insiste sur la pertinence typologique de la resyllabation (Cao, 1985: 298). Mais cette pertinence n'implique pas comme il le soutient que la linéatité ait un statut radicalement différent dans les langues qui ignorent resyllabation (et, étant de type « monosyllabique », ignorent aussi le PAM).

6. Dans la présente approche on ne peut plus supposer le sens de la syllabation variable.

7. Un effet spécifique de la construction régressive est l'asymétrie suffixes/préfixe (Sauzet, 1998).

8. En accord donc avec des approches comme celle de Lieber (1992) et à l'encontre d'une thèse centrale de la morphologie distribuée qui unit par règle contenu non phonique et réalisation phonique au moins pour les affixes grammaticaux. Poser des morphèmes dotés d'un contenu phonique dès le départ garantit l'effet des conditions de localité sur les opérations morphologiques assumées par la morphologie distribuée. Reste à montrer que le format de morphèmes utilisé ici permet de se dispenser des opérations intervenant en amont de l'insertion. 9. $a-b=b-a$ et $a-b, a-c$ n'implique pas que $c=a(n i$ même $a-b$, $a-c$ et $a-d$ que $d=b$ ou $d=c$ ). 10. Elle n'est plus « atomique» comme le maintient Chomsky (1995).

11. En fait, on peut supposer que l'attenance associe initialement des éléments au sens de Kaye, Lowenstamm \& Vergnaud (1985) et des travaux ultérieurs de la phonologie du gouvernement. En utilisant ici des segments je suppose donc une première résolution accomplie. Il faudra reprendre à partir d'une caractérisation élémentaire les cas de latences multiples où les divers segments latents ne diffèrent que par un trait ou un élément. En posant ici plusieurs segments, on laisse inexprimée la ressemblance assez générale des segments latents associés. Elle s'explique évidemment historiquement mais peut rester pertinente synchroniquement.

12. Reformulant un modèle déjà utilisé dans (Sauzet, 1994a).

13. C'est une nécessité décisive dans la forme de berbère analysée dans Dell \& Elmedlaoui $(1985,1988)$ réinterprétée selon la perspective présente dans (Sauzet, 1994a : 319).

14. En fait si [1] n'est pas syllabique en surface en français, il faut sans doute poser qu'il l'est abstraitement comme en occitan (Sauzet, 1994a : 167).

15. La présente analyse est neutre par rapport à la notion de chemin apophonique (Ségéral, 1995), (Scheer \& Ségéral, 1996).

16. Les choses se formulent peut-être plus clairement si on admet des formes lexicales plus connexes du type suivant :

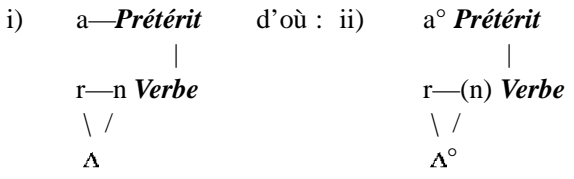

où la concurrence de $\Lambda^{\circ}$ et de $\mathrm{a}^{\circ}$ apparaît mieux. 
17. Il reste à montrer de manière étendue comment l'ensemble des effets d'une morphologie concaténative peut être obtenu selon les perspectives esquissées ici. Sauzet (1994b) envisage la question du ressaut en arabe moderne, où l'interdépendance phonologique d'effet concaténatifs et non concaténatifs s'intègre bien aux propositions que je défends.

18. En relevant une classe relativement restreinte de couples de morphèmes masculin/féminin, Morin (1983: 142) ne propose pas une résolution morphologique de la latence. En fait la question

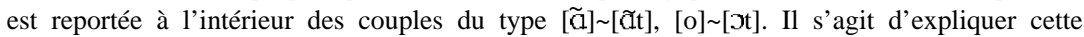
alternance comme celle des radicaux. Y.-Ch. Morin associe aux faits de latence des alternances de segments réalisées [œr [øz] que je propose dans la suite de cet article de traiter de manière semblable aux faits de latence.

19. À l'exception des consonnes nasales (cette lacune sera discutée plus loin) et de /1/. Dell (1973).

20. Et aussi des formes antérieures du français puis qu'on admet en général que les effacements de consonnes finales remontent au XIII ${ }^{e}$ siècle et la chute de schwa au XVII ${ }^{e}$ siècle.

21. Pour les analyses par supplétion voir (Schane, 1978), (Tranel, 1981), (Rotenberg, 1978) et de nombreuses autres références dans (Encrevé, 1988b).

22. Joel Rotenberg en proposant que les consonnes latentes sont lexicales, mais « à part», fait aussi le rapprochement avec les verbes forts (Rotenberg, $1978: 130$ ).

23. Dans le programme minimaliste, l'évaluation des dérivations ne se fonde que sur son déroulement et non sur son résultat (Chomsky, 1995 : 224). Je renvoie à la poursuite de la présente recherche la question de savoir si l'optimalité restreinte évoquée ici concerne les dérivations elles-mêmes ou leurs résultats.

24. Cette analyse de la liaison repose sur la resyllabation. Une thèse centrale de Pierre Encrevé est que l'existence de la liaison non enchaînée exige de dissocier resyllabation et liaison (Encrevé, 1988a). Il me semble que la syllabation est directement la seule manière de fonder la liaison, et que la liaison non enchaînée relève d'une explication secondaire (resyllabation parasitique).

25. Devant une consonne le résultat est le même que l'adjectif connaisse ou non une syllabation autonome. Comparez : « divin Télémaque », « malin génie ».

26. Un problème se pose ici. Si «blanc» prénominal est actif, «blanc ananas » est semblable à «malin esprit». Morin ne relève pas de réalisation de consonne nasale entre l'adjectif et le nom. Elle ne me parait pas exclue, mais cette absence pose problème. Toutefois, autant la consonne nasale après la voyelle nasale est obligatoire après les déterminants « un», «mon»... autant elle n'est pas absolument requise après un adjectif. En particulier toute insistance sur l'adjectif permet de la suspendre. Pour un adjectif qui n'est pas régulièrement prénominal, la resyllabation pourra être supposée bloquée par une diction plus contrôlée, aussi bien pour «blanc-(n)-ananas », que pour « coquin-(n)-ananas » ou « débrouillard-(r)-ananas », bien moins volontiers resyllabé que « fort-r-accent», voir (32 c). Dans le cas de /t/ («franc-t-ananas »), supposé inerte, il ne s'agit pas de resyllabation mais de syllabation d'où l'absence de blocage.

27. Cette dualité remonte ici à un processus historique de dévoisement devenu inactif en français standard. Si on raisonne en terme d'éléments (voir note 7, ci-dessus) et non de segments, il faudrait sans doute pose un élément dissocié correspondant au voisement (ou à son absence).

28. Dans une forme comme «vert » [ver], «verte », «verdure »... il faut supposer que $[\mathrm{t}]$ est associé à la catégorie adjectif.

29. En fait l-e-Dét est déjà marqué pluriel. On peut envisager que l'article défini est un morphème complexe du type :

i) 1-כ-Dét-Plur-e

/e/ est mobilisé en contexte pluriel et évité en contexte singulier.

30. Éventuellement si le déterminant n'est pas inerte (le) est formé parallèlement. 
31. Dans l'esprit d'une économie de dérivation ou de mouvement minimal du programme minimaliste (Chomsky, 1995) ou, si l'on évalue sur le résultat, par des considérations de marque syllabique que l'optimalité enregistre par une contrainte «éviter les codas» (McCarthy \& Prince, 1997).

32. Dell (1973: 180) note leur existence mais renvoie leur traitement à plus tard. Logiquement les tenants d'une analyse morphologique mettent davantage ces faits en avant, ainsi Morin (1992 : 239).

33. Elle permet aussi de représenter les alternances vocaliques, comme suggéré en (18), (19), (20) pour une forme apophonique de conjugaison anglaise. On peut traiter ainsi en français les alternances du type "fleur, fleuriste, floral » et celle des verbes, qu'elles soient liées à l'accentuation ou au temps. De l'interaction de la fusion de la voyelle temporelle et de la latence consonantique découlent les alternances typiques du prétérit français : «sais, savons »vs «sus». La place manque pour traiter ces formes ici.

\section{RÉFÉRENCES}

Abeillé, Anne; Godard, Danielle (1999).La position de l'adjectif épithète en français: le poids des mots (dans ce volume).

BAKER, Marc (1988). Incorporation : a theory of Gramatical Function Changing, Chicago : University of Chicago Press.

BAKER, Mark (1985). The Mirror Principle and Morphosyntaxic Explanation. Linguistic Inquiry $16: 373-415$.

CAO, Xuan Hao (1985). Phonologie et linéarité : réflexions critiques sur les postulats de la phonologie contemporaine, Paris : Selaf.

Сномsкy, Noam (1995). The Minimalist Program, Cambridge (Mass.)/Londres : MIT Press.

Clements, George N. ; Keyser, Samuel Jay (1981). A Three-Tiered Theory of the Syllable. MIT, Center for Cognitive Studies, Occasional Paper 19.

CoRnulier, Benoît de (1982). Théorie du vers, Rimbaud, Verlaine, Mallarmé, Paris : Seuil.

Dell, François (1973). Les Règles et les Sons, introduction à la phonologie générative, Paris : Hermann. (rééd. 1985)

Dell, François ; ElmedlaouI, Mohamed (1985). Syllabic Consonant and Syllabification in Imdlawn Tashlhiyt Berber. Journal of African Languages and Linguistics 7 : 105-130.

Dell, François ; Elmedlaoui, Mohamed (1988). Syllabic Consonants in Berber : Some New Evidence. Journal of African Languages and Linguistics 10 : 1-17.

DURAND, Marguerite (1936). Le Genre gramatical en français parlé, Paris : D'Artrey.

ENCREVÉ, Pierre (1988a). La Liaison avec et sans enchaînement, Paris : Seuil.

ENCREVÉ, Pierre (1988b). La liaison en français dans la phonologie générative. Recherches Linguistiques de Vincennes 17 : 55-108.

Halle, Morris ; Marantz, Alex (1993). Distributed Morphology and the Pieces of Inflexion in Kenneth Hale \& Samuel Jay Keyser (eds.), The View from Building 20, Essays in Linguistics in Honor of Sylvain Bromberger, Cambridge (Mass.)/Londres : MIT Press, 111-176.

HocketT, Charles (1955). A manual of phonology, Baltimore : Waverly Press.

ITô, Junko (1986). Syllable theory in prosodic Phonology, Ph. D. Diss. University of Massachusetts.

ITô, Junko (1989). A prosodic theory of epenthesis. Natural Languages and Linguistic Theory 7 : 217-159.

KaYe Jonathan D. ; Lowenstamm, Jean (1984). De la syllabicite.Dans Dell, Hirst \& Vergnaud (eds), Forme Sonore du Langage, 123-159. 
KAYE, Jonathan D. ; Lowenstamm, Jean ; Vergnaud, Jean-Roger (1985). The internal structure of phonological elements : a theory of charm and government.Dans Phonology Yearbook, 305-328. (Trad. française dans Recherches linguistiques de Vincennes 17, 1988, 109-134).

KAYNE, Richard (1994). The Antisymmetry of Syntax, Cambridge (Mass.)/Londres : MITPress.

LAFONT, Robert ; GARDÈS-MADRAY, Françoise (1976). Introduction a l'analyse textuelle, Paris : Larousse.

LeVIn, Juliette (1985). A Metrical Theory of Syllabicity, Ph. D. Diss., MIT.

LIEBER, Rochelle (1980). The organization of the lexicon, Ph. D. Diss. MIT[aussi: IULC 1981 ; New York/Londres: Garland 1990].

LIEBER, Rochelle (1992). Deconstructing Morphology, Chicago : University of Chicago Press.

Marantz, Alec (1997). No escape from syntax: don't try Morphological analysis in the privacy of your own Lexicon.Dans Dimitriadis, Siegel et al. (eds), University of Pennsylvania Working Papers in Linguistics 4.2 (Proceedings of the $21^{\text {st }}$ Annual Penn Linguistics Colloquium) 201-225.

McCarthy, John J. (1982). Formal Problems in Semitic Phonology and Morphology, Bloomington: IULC.

McCarthy, John J. ; Prince, Alan S. (1997). L'émergence du non marqué. L'optimalité en morphologie prosodique. Langages 125 : 55-99.

Morin, Yves-Charles (1983). De la (dé)nasalisation et de la marque du genre en français, Lingua 61, 2/3: 133-156.

Morin, Yves-Charles (1992). Un cas méconnu de la déclinaison de l'adjectif français: les formes de liaison de l'adjectif antéposé.Dans André Clas (ed), Le Mot, les Mots, les Bons Mots. Word, Words, Witty Words. Hommage à Igor Mel'cuk, Montréal: Presses de 1'Université de Montréal, 233-250.

Rotenberg, Joel (1978). The Syntax of Phonology, Ph. D. Diss. MIT.

SAUZET, Patrick (1994a). Attenance, gouvernement et mouvement en phonologie. Les constituants dans la phonologie et la morphologie de l'occitan, Montpellier : CEO-UPV. [thèse Paris8, 1993]

SAUZET, Patrick (1994b). Extensions du modèle a-linéaire en phonologie : syllabe, accent, morphologie, Mémoire pour 1'habilitation à diriger des recherches, Université de Paris VII.

SAUZET, Patrick (1996). Ordre des mots, ordre dans les mots. Langue française 111 : 10-37.

SAUZET, Patrick (1998a). « Enamourer », « enivrer » et « enorgueillir »: le statut des préfixes. Dans Patrick Sauzet (ed), Langues et Grammaire II \& III : Phonologie. Département SDLParis 8: 117-140.

SAUZET, Patrick (1998b). Clise et liaison (communication au Colloque La cliticisation, Bordeaux 23-26 septembre 1998).

Schane, Sanford (1965). The Phonological and Morphological Structure of French. Ph. D. Diss. MIT.

Schane, Sanford (1968). French Phonology and Morphology, Cambridge Massachusetts : MIT Press.

Schane, Sanford (1978). Deletion vs Epenthesis : A Pseudo controversy. Studies in French Linguistics 1 (2) : 71-78.

SEgui, Juan, (1992). Perception du langage et modularité. Dans Daniel Andler (ed), Introduction aux sciences cognitives, 131-152

SCHEER, Tobias ; SÉGÉRAL, Philippe (1996). L'apophonie dans les verbes forts de l'allemand moderne. ms Paris 7.

SÉGÉRAL, Philippe (1995). Une théorie généralisée de l'apophonie, Thèse Paris 7.

SELKIRK, Elisabeth (1982). The Syntax of Words, Cambridge (Mass.): MIT Press. 
SPENCER, Andrew (1991). Morphological theory : an introduction to word structure in generative grammar, Oxford : B. Blackwell.

TRANEL, Bernard (1981). Concreteness in generative phonology : evidence from French, Berkeley : University of California Press.

Vergnaud, Jean-Roger (1982). On the foundation of phonology. Communication au colloque de GLOW(Paris). (inédit)

WAuQuier-Gravelines, Sophie (1999). Segmentation lexicale de la parole continue : la linéarité en question. (dans ce volume)

Williams, Edwin (1981). On the Notions "Lexically related" and "Head of a Word". Linguistic Inquiry 12-2 : 245-274.

\begin{abstract}
Suppose linearity doesn't pre-exist to syllabification but is built by it, within morphemes, on the basis of the relative accessibility of segments. On the one hand, applying syllabification to syntacticlike structures allows deriving, via phonology, part of the order prevailing between morphemes. Morphology and words thus appear as effects of phonology. On the other hand, it makes it possible for morphemes to embody a segmental content more connected than traditional concatenated morphemes are, or, on the contrary, presenting discontinuities. Elaborating upon this latter possibility, latent consonants (as are typically present in the phonology of French) are analysed as dissociated segments, viz. dissociated from the bulk of the phonetic specifications (instead of « floating » which, it is claimed, appears to be more problematic than is currently assumed). The analysis advocated here not only allows dealing with invariable latency (« petit, petit-t-ami, petitesse »), but also with variable latency (« grand, grande, grand-t-ami », « vert, verte, verdure »), suppletion (« beau, bel ») and other word final variability phenomena (« sec, sèche »).
\end{abstract}

\title{
KEYWORDS
}

Linearity, latent consonants, « liaison », syllabification, French. 